\title{
Cigarette smoke extract induces migration of rat vascular smooth muscle cells through regulation of expressions of MOB2 and LATS1
}

\author{
Jing Zhang ${ }^{1,2}$, Wei Zhou ${ }^{3}$, Shenghu He ${ }^{2}$, Yang Qian ${ }^{2,4}$, Daxin Wang ${ }^{1 *}$ \\ ${ }^{1}$ School of Medicine, Yangzhou University, ${ }^{2}$ Department of Cardiology, Northern Jiangsu People's Hospital, ${ }^{3}$ Department of \\ Hematology and Rheumatology, Affiliated Hospital of Yangzhou University, ${ }^{4}$ Dalian Medical University, Yangzhou City, China
}

*For correspondence: Email: pduzq8@163.com

Sent for review: 17 December 2020

Revised accepted: 3 March 2021

\begin{abstract}
Purpose: To study the impact of cigarette smoke extract (CSE) on the migratory capacity of rat vascular smooth muscle cells (VSMCs), and the likely mechanism of action involved.

Methods: Rat VSMCs were exposed to varying concentrations of CSE. The migration capacity of the cells was determined by Transwell migration assay and scratch test. Protein and RNA expression levels of LATS1 (large tumor suppressor kinase 1) and MOB2 (monopolar spindle-one-binder protein 2) were assayed using immunoblotting and quantitative real-time-polymerase chain reaction (qRT-PCR), respectively.

Results: The results showed that within a certain concentration range, the migratory capacity of VSMCs increased as the concentration of CSE increased $(p<0.05)$. In contrast, the protein and RNA expressions of LATS1 and MOB2 were negatively correlated with CSE concentrations $(p<0.05)$.

Conclusion: CSE promotes abnormal migration of rat VSMCs by inhibiting the expressions of LATS1 and MOB2 associated with Hippo signaling pathway. Thus, smoking may induce vascular remodeling and cardiovascular events via this mechanism.
\end{abstract}

Keywords: Cigarette smoke extract, Migration, Vascular smooth muscle cells, MOB2, LATS1

\begin{abstract}
This is an Open Access article that uses a fund-ing model which does not charge readers or their institutions for access and distributed under the terms of the Creative Commons Attribution License (http://creativecommons.org/licenses/by/4.0) and the Budapest Open Access Initiative (http://www.budapestopenaccessinitiative.org/read), which permit unrestricted use, distribution, and reproduction in any medium, provided the original work is properly credited.
\end{abstract}

Tropical Journal of Pharmaceutical Research is indexed by Science Citation Index (SciSearch), Scopus, International Pharmaceutical Abstract, Chemical Abstracts, Embase, Index Copernicus, EBSCO, African Index Medicus, JournalSeek, Journal Citation Reports/Science Edition, Directory of Open Access Journals (DOAJ), African Journal Online, Bioline International, Open-J-Gate and Pharmacy Abstracts

\section{INTRODUCTION}

Coronary heart disease (CHD) is a primary disease which seriously threatens human health and causes mortality worldwide. With improvements in living standards, the incidence of CHD has noticeably increased. It is generally believed that smoking contributes significantly to incidents of CHD. The global report of the World Health Organization (WHO) on Prevalence of
Tobacco Use (2000 - 2025) stated that smoking and passive smoking are important factors that predispose to severe cardiovascular diseases (CVDs). About 3 million CVD patients annually die because of smoking or passive smoking [1].

It has been shown that vascular remodeling is a shared pathophysiological process which leads to CVDs such as coronary heart disease, in-stent re-stenosis following percutaneous coronary 
intervention, and hypertension [2]. Furthermore, abnormal proliferation and migration of vascular smooth muscle cells (VSMCs) to the subintimal space are important pathological bases of vascular remodeling [3]. However, the involvement of smoking in the pathogenesis of vascular remodeling, and its effect on the proliferative and evasive potential VSMCs, and the specific biological processes involved, remain to be investigated.

The Hippo signal route is a conservative pathway which was recently discovered. This pathway is implicated in the formation of the heart and vessels, damage response, cardiac regeneration and angiogenesis [4]. Dysfunction of the Hippo signaling pathway may lead to incidents of CVDs such as acute myocardial infarction, myocardial hypertrophy, intimal hyperplasia, and arteriosclerosis [5]. Monopolar spindle-onebinder protein 2 [MOB2] and large tumor suppressor gene 1 [LATS1] are key factors associated with the Hippo signaling pathway, and they are closely linked with abnormal cell migration [6].

In the present study, VSMCs were exposed to cigarette smoke extract (CSE), and Transwell migration assay and scratch test were used to determine the influence of the treatment on cellular migration. Protein and RNA expressions of LATS1 and MOB2 were assayed with immunoblotting and RT-qPCR, respectively. The mechanism by which CSE induced vascular remodeling was also determined, so as to identify the therapeutic targets.

\section{EXPERIMENTAL}

\section{Reagents}

Antibodies for MOB2 (\#SAB1301138) were produced by Merck.; LATS1(\#3477), anti-rabbit antibody (\#7074) and GAPDH (\#5174) were bought from Cell Signaling Technology (Danvers, MA, USA). Fetal bovine serum (FBS) and Dulbecco's modified Eagle's medium (DMEM) were obtained from Hyclone Laboratories Inc (Logan, UT, USA), while PVDF membranes were products of Merck Millipore (Burlington, MA, USA). Substrate kits for ECL were produced by TFSc (Waltham, MA). Hongjinlong cigarette was purchased from Hubei Tobacco Industrial Co. Ltd (Wuhan, China).

Radioimmunoprecipitation assay (RIPA) lysis buffer and BCA Kits were supplied by Beijing Solarbio Science \& Technology Co. Ltd, Beijing, while RNA extraction kit, cDNA synthesis kit and
PCR kit were purchased from TaKaRa (Kyoto, Japan).

\section{Instruments}

Tecan Infinite M2000 microplate reader was purchased from Tecan Group Ltd. (Männedorf, Switzerland). ChemiDoc XRS+ imaging system was obtained from Bio-Rad, while RT-PCR equipment was purchased from $A B$ (Foster City, CA, USA).

\section{Preparation of CSE}

The CSE was prepared as follows: One cigarette containing $10.0 \mathrm{mg}$ of tar and $0.9 \mathrm{mg}$ of nicotine was lit. Then, a $50-\mathrm{mL}$ syringe was used to draw cigarette smoke into a sealed flask containing 20 $\mathrm{mL}$ phosphate-buffered saline (PBS) solution for 3 - 5 min until the cigarette burned out. Then, the solution was passed through a $0.22-\mu \mathrm{m}$ filter to remove any bacteria. The product obtained was a $100 \%$ stock solution of CSE.

\section{Cell culture}

Rat VSMCs were donated by the Department of Pathophysiology of Nanjing Medical University (Nanjing, China). All animal experiments were undertaken in accordance with standard guidelines for cell culture research. This investigation received approval from the Animal Use and Care Committee of our institution (approval no. 20200639). The cells were cultured in complete medium at $37^{\circ} \mathrm{C}$ in an incubator with $5 \% \quad \mathrm{CO}_{2}$. They were subjected to tryptic digestion and passage, and those at logarithmic phase within 10 generations and good growth status were harvested for subsequent experiments.

Grouping: The cells were divided into groups exposed to different concentrations of CSE. Cells in the blank control were treated for $12 \mathrm{~h}$ with complete medium without CSE. Cells in the low-, medium-, and high-CSE groups were cultured in complete medium containing 1,5 , and $10 \%$ CSE, respectively, for $12 \mathrm{~h}$ [7-9].

\section{Determination of two-dimensional (2D) migration capacity of VSMCs using scratch test}

The VSMCs were suspended in DMEM containing $2 \%$ FBS, and inoculated onto a 6-well plate. When the cells grew to 80 - $90 \%$ confluency, a pipette tip was used to scratch the center of each well. They were maintained in DMEM spiked with $2 \%$ fetal bovine serum at 37 ${ }^{\circ} \mathrm{C}$ for $24 \mathrm{~h}$ in a $5 \% \mathrm{CO}_{2}$ incubator, and their 
images were taken at 0,24 , and $48 \mathrm{~h}$. The mean scratch width was calculated as an index of cell migration.

Transwell migration assay of three-
dimensional
VSMCs

A suspension of rat VSMCs was inoculated in a 6 -well plate $(2 \mathrm{~mL} /$ well). Further culturing was carried out for $12 \mathrm{~h}$, and the cells were digested with trypsin, followed by cell counting. Cell concentration was maintained at $2 \times 10^{5}$ cells $/ \mathrm{mL}$. Thereafter, the suspension $(200 \mu \mathrm{L})$ diluted with serum-free DMEM was transferred to the top compartment, while each well of the chamber beneath contained $500 \mu \mathrm{L}$ of medium. After further cultivation for $24 \mathrm{~h}$, the cells were fixed in formaldehyde and stained with crystal violet. Cells in the upper chamber were wiped off, and cells in the lower chamber were counted microscopically. For each chamber, 3 fields of view were randomly chosen for statistical analysis. Each test was carried out in triplicate.

\section{Assay of protein expression levels of MOB2 and LATS1}

Protein extraction was carried out with RIPA buffer in strict accordance with the manufacturer's instructions. In this method, 200 $\mu \mathrm{L}$ of RIPA lysis buffer containing $10 \%$ protease inhibitor was added to each plate. After lysing for $10 \mathrm{~min}$ on ice, the cells were centrifuged at $12000 \mathrm{rpm}$ for $10 \mathrm{~min}$ at $4{ }^{\circ} \mathrm{C}$, and the supernatant was collected. The content of each target protein was measured using BCA method. The protein samples were thoroughly mixed with $5 x$ protein loading buffer and denatured in boiling at $100{ }^{\circ} \mathrm{C}$ for $10 \mathrm{~min}$. The denatured protein samples were then preserved at $-20^{\circ} \mathrm{C}$. Equal amounts of proteins (30 $\mathrm{\mu g}$ each) were resolved using SDS-polyacrylamide gel electrophoresis, followed by transfer onto PVDF membranes which were then sealed with $5 \%$ defatted milk powder for $2 \mathrm{~h}$. Then, the membrane was incubated with primary antibodies, i.e. MOB2 antibody (1:1000), LATS1 antibody (1:1000), and GAPDH $(1: 1000)$ at $4^{\circ} \mathrm{C}$ overnight. Thereafter, the membrane was rinsed thrice with PBS containing $0.1 \%$ Tween-20 (PBST), followed by incubation with the corresponding HRPconjugated secondary antibodies at the room temperature for $1 \mathrm{~h}$. Finally, the membrane was washed thrice with PBST, and the grayscale values of each band were analyzed using the ChemiDoc XRS+ imaging system, with GAPDH as internal reference. Protein expression levels were determined in relation to that of the standard gene (GAPDH).

\section{Assay of mRNA expression levels of MOB2 and LATS1}

Primers were designed for each target gene and internal reference gene GAPDH using Primer Premier 5.0. The primers were synthesized by Sangon Biotech Co. Ltd. (Shanghai, China). The primer sequences are shown in Table 1.

Table 1: The primer sequences used in PCR

\begin{tabular}{lll}
\hline Gene & & Primer sequence \\
\hline LATS1 & upstream & 5'- \\
& & AGCTCACTCTCTGGTTG \\
& & GGA-3' \\
& downstream & 5'- \\
& & CTTGGCTTGAGGTGGG \\
& & ATGT-3' \\
MOB2 & upstream & 5'- \\
& & CCCAAGCTTCCATGGA \\
& & CTGGCTCATGGGGAAG \\
& & TC-3' \\
& \multirow{2}{*}{ downstream } & 5'- \\
& & CGGGGTACCTCATCTC \\
& & CTTCACGTGGTTCTG-3' \\
GAPDH & upstream & 5'- \\
& & TGGCAAAGTGGAGAT \\
& & GTTGCC-3' \\
& downstream & 5'- \\
& & AAGATGGTGATGGGCT \\
& & TCCCG-3' \\
\hline
\end{tabular}

Following extraction of total RNA, cDNA was synthesized using cDNA synthesis kit, followed by addition of SYBR Green-I. The RT-qPCR was done using StepOnePlus Real-Time PCR System. Amplification curve and dissolution curve were plotted, and CT values of each target gene were calculated. The standard gene used was GAPDH. Relative mRNA levels of the target genes were determined using $2^{-\triangle \triangle}{ }^{\mathrm{CT}}$ procedure.

\section{Statistical analysis}

Measured data are presented as mean \pm SD. Inter-group comparison was done with one-way ANOVA, while pairwise comparisons were done with q-test. Tukey's post hoc test was used with one-way ANOVA. All analyses were done with SPSS 20.0 software. Values of $p<0.05$ were considered statistically significant.

\section{RESULTS}

\section{Effect of different concentrations of CSE on 2D migration capacity of rat VSMCs}

Scratch width at each CSE concentration group was markedly smaller than the negative control value, while the degree of width narrowing was more pronounced. The scratch width was narrowed as concentrations of CSE increased, 
and the degree of narrowing was markedly higher in $10 \%$ CSE group than in the negative control group $(p<0.05)$. Moreover, narrowing in $10 \%$ CSE cells was markedly increased, relative to $1 \%$ CSE cells. In general, narrowing increased with increase in CSE concentration $p<$ 0.05). These results are shown in Figure 1.

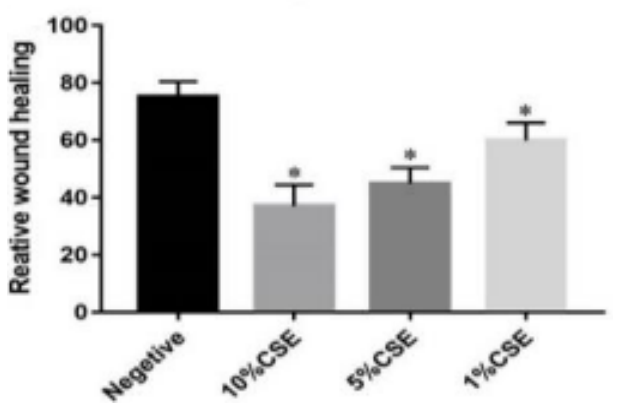

Figure 1: Effects of different concentrations of CSE on 2D migration capacity of rat VSMCs. Scratch width in each CSE concentration group was significantly smaller than that in the negative control group, and the degree of narrowing was higher. As the concentration of CSE was increased, the scratch width became narrower, and the degree of narrowing was markedly increased. ${ }^{*} P<0.05$, compared with -ve control

\section{Effect of different concentrations of CSE on 3D migration capacity of rat VSMCs}

There was a significant increase in the population of VSMCs in the lower compartment in each CSE concentration group, relative to negative control value. As concentrations of CSE increased, the number of rat VSMCs migrating to the lower chamber was markedly increased. These results are presented in Figure 2 and Figure 2.

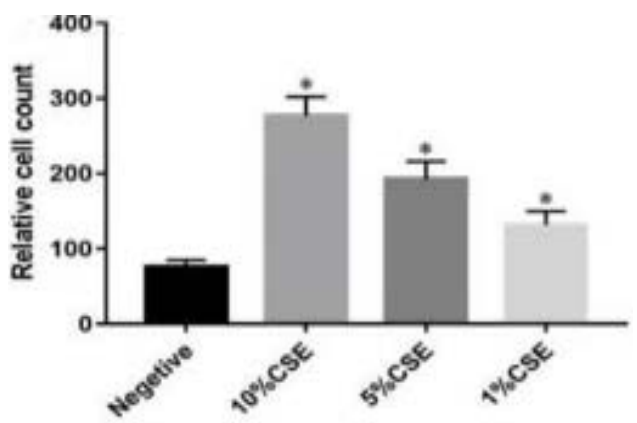

Figure 2: Effect of different concentrations of CSE on 3D migration capacity of rat VSMCs. There was marked increase in the population of rat VSMCs that moved to the lower compartment at each CSE concentration group, relative to negative control value. With increase in concentration of CSE, the population of rat VSMCs that moved to the lower compartment increased noticeably. ${ }^{*} P<0.05$, vs. negative control
Effect of different concentrations of CSE on LATS1 and MOB2 protein expressions in VSMCs

The protein expression levels of LATS1 and MOB2 in rat VSMCs at each CSE concentration were significantly decreased, when compared with those in the negative control group. The protein expression levels of LATS1 and MOB2 increased with increase in CSE concentration, as shown in Figure 3 and Figure 4.

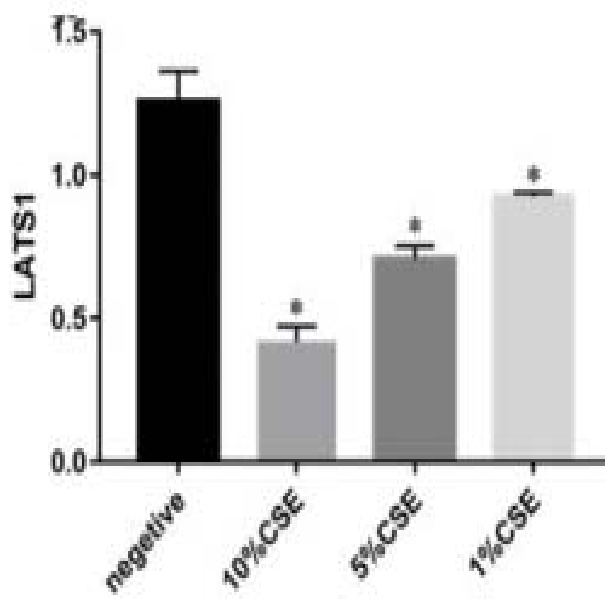

Figure 3: Influence of different concentrations of CSE on LATS1 protein expression in rat VSMCs. The LATS1 protein expression in rat VSMCs at each CSE concentration group was markedly reduced, relative to negative control. With increase in concentration of CSE, the LATS1 protein expression was markedly reduced

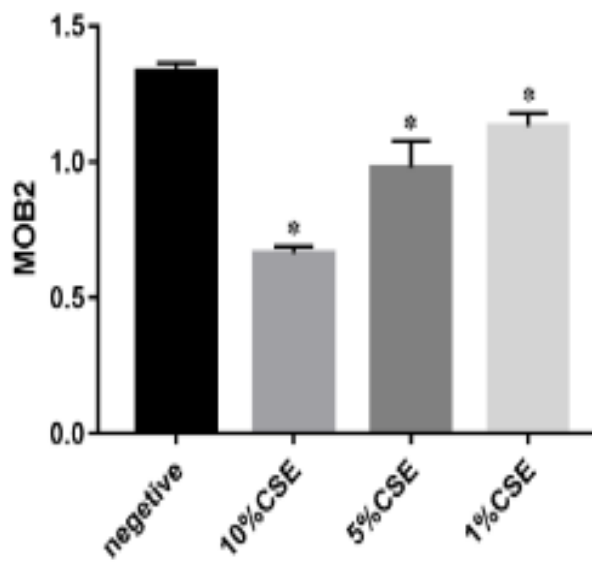

Figure 4: Influence of different concentrations of CSE on MOB2 protein expressions in rat VSMCs. The MOB2 protein expression in rat VSMCs in each CSE concentration group was markedly reduced, relative to negative control. With increase in concentration of CSE, the MOB2 protein expression was significantly reduced 
Impact of different concentrations of CSE on mRNA expressions of LATS1 and MOB2 in rat VSMCs

The mRNA expression levels of LATS1 and MOB2 in rat VSMCs at each CSE concentration were significantly attenuated, relative to negative control values. The mRNAs of LATS1 and MOB2 reduced in concentration with increase in the concentration of CSE. These results are shown in Figure 5 and Figure 6.

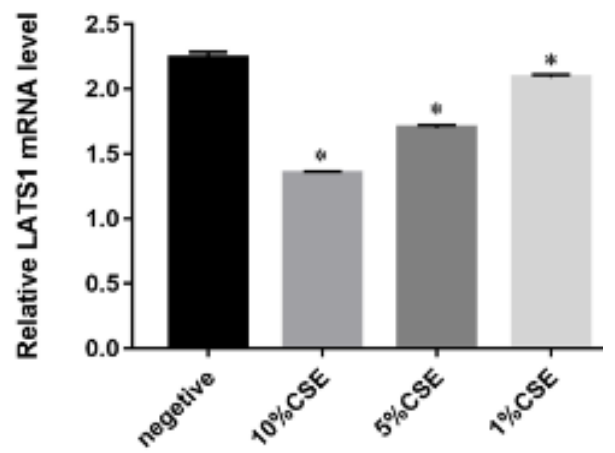

Figure 5: Influence of different concentrations of CSE on mRNA expression levels of LATS1 in rat VSMCs. The LATS1 mRNA expression in rat VSMCs in each CSE concentration group was significantly attenuated, relative to negative control value. As the concentration of CSE increased, the LATS1 mRNA expression was markedly decreased

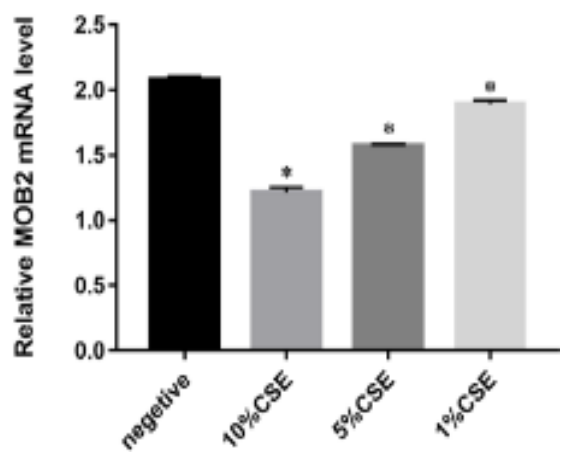

Figure 6: Effect of different concentrations of CSE on MOB2 mRNA expression in rat VSMCs. The MOB2 mRNA expression in rat VSMCs in each CSE concentration group was markedly attenuated, relative to the negative control value. As the concentration of CSE increased, the MOB2 mRNA expression level markedly decreased. ${ }^{*} P<0.05$, vs. negative control group

\section{DISCUSSION}

Cardiovascular diseases (CVDs) are a leading cause of death globally. Factors such as improvements in lifestyle, pressure of work, changes in dietary habits, hypertension, atherosclerosis, and in-stent re-stenosis after percutaneous coronary intervention, have seriously threatened human health. Thus, an understanding of the pathophysiology of CVDs is highly essential for developing novel therapeutic strategies for these diseases. The risk factors for CVDs include age, gender, genetics, diabetes, disorders in lipid metabolism, and smoking. Indeed, smoking is recognized as a primary risk factor for CVDs [10]. In recent years, researchers have focused attention on the correlation between smoking and CVDs. However, the correlation has not yet been fully understood.

Vascular remodeling is closely associated with the occurrence of CVDs. Vascular remodeling refers to structural changes in at least four cellular processes: cell growth, cell death, cell migration, and synthesis or degradation of extracellular matrix (ECM). It is characterized by endothelial dysfunction, proliferation and migration of VSMCs, and ECM deposition [11]. As integral components of the vascular wall, VSMCs enhance structural support to vascular wall, and regulate vascular tone and vascular remodeling. However, VSMCs play dual roles. On the one hand, they promote vascular growth and repair, while on the other hand, they may lead to CVDs such as atherosclerosis, intimal hyperplasia, and re-stenosis. Therefore, abnormal proliferation and migration of VSMCs play a crucial role in vascular remodeling. An understanding of the pathological mechanism involved in abnormal proliferation and migration of VSMCs in the early stage of vascular remodeling is significant for arresting vascular remodeling, in addition to early control and treatment of CVDs.

In agreement with the findings in this investigation, it has been reported that smoking promotes abnormal proliferation and migration of VSMCs [12]. The scratch width at each CSE concentration was markedly smaller than the negative control value, while the degree of narrowing of scratch width was higher. With increase in CSE concentration, the scratch width became narrower, and the degree of merging was significantly higher. There was a marked increase in the population of VSMCs that moved to the lower compartment at each CSE concentration group, relative to negative control value. As CSE concentrations increased, the number of VSMCs migrating to the lower chamber also increased. In Transwell migration assay and scratch test, results showed that within a certain concentration range, smoking promoted excessive VSMC migration, thereby facilitating vascular remodeling and CVDs. 
The Hippo signaling pathway which was first discovered in the fruit fly, controls organ size in animals through regulation of cell proliferation and apoptosis. Studies have revealed the involvement of this pathway in the formation of the cardiovascular system, including developmental differentiation of heart and vessels, damage response, cardiac regeneration and angiogenesis [13]. Impairment of the Hippo signaling pathway may lead to incidents of cardiovascular diseases such as myocardial infarction, myocardial hypertrophy, vascular intimal hyperplasia and arteriosclerosis [14]. A key factor related to the Hippo signaling pathway in mammals is LATS1 which is localized at chromosome 6q25.1. The MOB2 gene is an evolutionarily conservative gene which possibly regulates how cells proliferate and differentiate [15]. The MOB2 protein binds to Cbk1, activates Cbk1 kinase and regulates cell polarity, cell cycle, and cell growth. The synergistic interaction among MOB2, Cbk1 and Orb6 are important for maintenance of cell polarity during mitosis and cell cycle [16]. However, the roles of LATS1 and MOB2 in the regulation of VSMCs have not been fully elucidated.

The findings of the present research indicate that mRNAs of LATS1 and MOB2 in rat VSMCs at each CSE concentration were appreciably decreased, relative to negative control values. As the concentration of CSE increased, the mRNA expression levels of LATS1 and MOB2 were markedly attenuated, indicating that LATS1 and MOB2 expressions were weakened as the migration capacity of cells increased. Thus, it can be speculated that overexpression of LATS1 and MOB2 may inhibit the migration of VSMCs, thereby inhibiting vascular remodeling. Moreover, the effect of overexpression of LATS1 and MOB2 on VSMCs was studied. It has been shown that the tumor suppressor gene LATS1 is downregulated in different tumors [17]. Previous research has revealed that overexpression of LATS1 in esophageal squamous carcinoma cell line EC109 inhibited the proliferative, migratory, and invasive potential of tumor cells [18]. Moreover, overexpression of LATS1 activates the Hippo signaling pathway, and inhibits proliferation and migration of some stem cells [19]. This indicates the significance of LATS1 and MOB2 in cell migration.

In the current study, it was found that as CSE concentration increased, the migratory capacity of rat VSMCs increased as well. Moreover, the expressions of MOB2 and LATS1 decreased as the migration capacity of the cells increased. However, the specific biological mechanism remains to be further investigated.

\section{CONCLUSION}

The results obtained in this research indicate that CSE promotes abnormal migration of VSMCs through a mechanism associated with downregulation of the expressions of MOB2 and LATS1 in the Hippo signaling pathway. Therefore, CSE influences the migration of rat VSMCs by regulating the expressions of MOB2 and LATS1 in Hippo signaling pathway. Consequently, MOB2 and LATS1 may be used as therapeutic targets in clinical practice. Abnormal migration of VSMCs may be inhibited by regulating the expressions of MOB2 and LATS1, thereby delaying vascular remodeling and reducing the occurrence of cardiovascular events.

\section{DECLARATIONS}

\section{Acknowledgement}

This study was financially supported by Six Talent Peak Research Project in Jiangsu Province (no. 2016-WSN-280); Key Talents of Science and Education in Yangzhou City in 13th Five-Year (no. ZDRC20189); and the Natural Science Foundation of Yangzhou City (no. YZ2019077).

\section{Conflict of interest}

No conflict of interest is associated with this work.

\section{Authors' contribution}

We declare that this work was performed by the authors named in this article and all liabilities pertaining to claims relating to the content of this article will be borne by the authors. Daxin Wang designed the study, supervised the data collection, and analyzed the data. Jing Zhang and Wei Zhou interpreted the data and prepared the manuscript for publication. Shenghu $\mathrm{He}$ and Yang Qian supervised the data collection, analyzed the data and reviewed the draft of the manuscript. Jing Zhang and Wei Zhou contributed equally to this work as co-first authors.

\section{Open Access}

This is an Open Access article that uses a funding model which does not charge readers or their institutions for access and distributed under the terms of the Creative Commons Attribution License (http://creativecommons.org/licenses/by/ 
4.0) and the Budapest Open Access Initiative (http://www.budapestopenaccessinitiative.org/rea d), which permit unrestricted use, distribution, and reproduction in any medium, provided the original work is properly credited.

\section{REFERENCES}

1. Mathers C, Stevens $G$, d'Espaignet $E T$, Wolfenden $L$. WHO global report: mortality attributable to tobacco. WHO 2012; 1-5.

2. Hao YM, Yuan HQ, Ren Z, Qu SL, Liu LS, Dang H, Yin K, Fu M, Jiang ZS. Endothelial to mesenchymal transition in atherosclerotic vascular remodeling. Clin Chim Acta 2019; 490: 34-38.

3. Chakraborty $R$, Saddouk $F Z$, Carrao AC, Krause DS, Greif DM, Martin KA. Promoters to study vascular smooth muscle. Arterioscler Thromb Vasc Biol 2019; 39: 603-612.

4. Byun J, Del Re DP, Zhai $P$, Ikeda S, Shirakabe $A$, Mizushima W, Miyamoto S, Brown JH, Sadoshima J. Yes-associated protein (YAP) mediates adaptive cardiac hypertrophy in response to pressure overload. J Biol Chem 2019; 294: 3603-3617.

5. John PL, Todd H, Min Z, Mahdis R, Yuka M, Matthew $\mathrm{CH}$, Ana S, James TW, James FM. Hippo pathway deficiency reverses systolic heart failure after infarction. Nature 2017; 550: 260-264.

6. Gundogdu R, Hergovich A. The possible crosstalk of MOB2 with NDR1/2 kinases in cell cycle and DNA damage signaling. J Cell Signal 2016; 1: 125.

7. Hu J, Xu YJ, Zhang ZX. Effect of cigarette smoke extract on proliferation of rat pulmonary artery smooth muscle cells and the relevant roles of protein kinase C. Chin Med J (Engl) 2007; 120(17). 1523-1528;

8. Li Y, Pu G, Chen C, Yang L. Inhibition of FHL1 inhibits cigarette smoke extract-induced proliferation in pulmonary arterial smooth muscle cells. Mol Med Rep 2015; 12(3): 3801-3808;

9. $\mathrm{Gu}$ Z, Fonseca V, Hai CM. Nicotinic acetylcholine receptor mediates nicotine-induced actin cytoskeletal remodeling and extracellular matrix degradation by vascular smooth muscle cells. Vascul Pharmacol 2013; 58(1): 87-97.
10. Mamudu HM, Paul T, Veeranki SP, Wang L, Panchal HB, Budoff M. Subclinical atherosclerosis and relationship with risk factors of coronary artery disease in a rural population. Am J Med Sci 2015; 350: 257-262.

11. Schiffrin EL. Vascular remodeling in hypertension: mechanisms and treatment. Hypertension 2012; 59: 367-374.

12. Guo T, Chai X, Liu Q, Peng W, Peng Z, Cai Y. Downregulation of $P 16$ promotes cigarette smoke extract-induced vascular smooth muscle cell proliferation via preventing G1/S phase transition. Exp Ther Med 2017; 14: 214-220.

13. Heallen $T$, Zhang $M$, Wang J, Bonilla-Claudio $M$, Klysik E, Johnson RL, Martin JF. Hippo pathway inhibits wnt signaling to restrain cardiomyocyte proliferation and heart size. Science 2011; 332: 458-461.

14. Artap S, Manderfield LJ, Smith CL, Poleshko A, Aghajanian $H$, See $K$, Li L, Jain R, Epstein JA. Endocardial Hippo signaling regulates myocardial growth and cardiogenesis. Dev Biol 2018; 440: 22-30.

15. Zhang W, Shen J, Gu F, Zhang $Y$, Wu W, Weng J, Liao $Y$, Deng $Z$, Yuan $Q$, Zheng $L$, et al. Monopolar spindleone-binder protein 2 regulates the activity of large tumor suppressor/yes-associated protein to inhibit the motility of SMMC-7721 hepatocellular carcinoma cells. Oncol Lett 2018; 15: 5375-5383.

16. Jorgensen $P$, Nelson $B$, Robinson $M D$, Chen $Y$, Andrews $B$, Tyers $M$, Boone $C$. High-resolution genetic mapping with ordered arrays of Saccharomyces cerevisiae deletion mutants. Genetics 2002; 162: 1091-1099.

17. Chen KH, He J, Wang DL, Cao JJ, Li MC, Zhao XM, Sheng $X, L i$ WB, Liu WJ. Methylation-associated inactivation of LATS1 and its effect on demethylation or overexpression on YAP and cell biological function in human renal cell carcinoma. Int J Oncol 2014; 45: 2511 2521.

18. Chai $Y$, Xiang $K, W u Y$, Zhang T, Liu Y, Liu X, Zhen W, Si $Y$. Cucurbitacin $B$ inhibits the Hippo-YAP signaling pathway and exerts anticancer activity in colorectal cancer cells. Med Sci Monit 2018; 24: 9251-9258.

19. Li L, Dong L, Wang Y, Zhang X, Yan J. Lats1/2-mediated alteration of Hippo signaling pathway regulates the fate of bone marrow-derived mesenchymal stem cells. Biomed Res Int 2018; 2018: 4387932. 